\title{
Multicenter study evaluating factors associated with treatment outcome for low back pain injections
}

\author{
Steven P Cohen 다 , ${ }^{1,2}$ Tina L Doshi $10,{ }^{1}$ Connie Kurihara, ${ }^{3}$ David Reece, ${ }^{3}$ \\ Edward Dolomisiewicz, ${ }^{3}$ Christopher R Phillips, ${ }^{4}$ Timothy Dawson, ${ }^{5}$ David Jamison, ${ }^{6}$ \\ Ryan Young, ${ }^{7}$ Paul F Pasquina ${ }^{3}$
}

\begin{abstract}
- Additional supplemental material is published online only. To view, please visit the journal online (http://dx.doi.org/ 10.1136/rapm-2021-103247).

${ }^{1}$ Department of Anesthesiology, Johns Hopkins School of Medicine, Baltimore, Maryland, USA

${ }^{2}$ Departments of Physical Medicine \& Rehabilitation and Anesthesiology, Walter Reed National Military Medical Center, Bethesda, Maryland, USA

${ }^{3}$ Department of Physical Medicine \& Rehabilitation, Walter Reed National Military Medical Center, Bethesda, Maryland, USA

${ }^{4}$ Department of Anesthesiology, Naval Hospital San Diego, San

Diego, California, USA ${ }^{5}$ Department of Anesthesiology, VA Puget Sound Health Care System Seattle Division, Seattle, Washington, USA

${ }^{6}$ Department of Anesthesiology, Walter Reed National Military Medical Center, Bethesda, Maryland, USA

${ }^{7}$ Department of Surgery, Landstuhl Regional Medical Center, Landstuhl Kirchberg, Rheinland-Pfalz, Germany
\end{abstract}

Correspondence to Dr Steven P Cohen, Anesthesiology, Johns Hopkins School of Medicine, Baltimore, MD 21205, USA:

scohen40@jhmi.edu

Received 11 October 2021 Accepted 10 November 2021 Published Online First 8 December 2021

\section{Check for updates}

(C) American Society of Regional Anesthesia \& Pain Medicine 2022. No commercial re-use. See rights and permissions. Published by BMJ.

To cite: Cohen SP Doshi TL, Kurihara C, et al. Reg Anesth Pain Med 2022:47:89-99.

\begin{abstract}
Background There has been a worldwide surge in interventional procedures for low back pain (LBP), with studies yielding mixed results. These data support the need for identifying outcome predictors based on unique characteristics in a pragmatic setting.
\end{abstract}

Methods We prospectively evaluated the association between over two dozen demographic, clinical and technical factors on treatment outcomes for three procedures: epidural steroid injections (ESIs) for sciatica, and sacroiliac joint (SIJ) injections and facet interventions for axial LBP. The primary outcome was change in patient-reported average pain intensity on a numerical rating scale (average NRS-PI) using linear regression. For SIJ injections and facet radiofrequency ablation, this was average LBP score at 1 and 3 months postprocedure, respectively. For ESI, it was average leg pain 1- month postinjection. Secondary outcomes included a binary indicator of treatment response (success).

Results 346 patients were enrolled at seven hospitals. All groups experienced a decrease in average NRS-PI $(p<0.0001$; mean 1.8 \pm 2.6$)$. There were no differences in change in average NRS-PI among procedural groups $(p=0.50)$. Lower baseline pain score (adjusted coefficient $-0.32,95 \% \mathrm{Cl}-0.48$ to $-0.16, p<0.0001)$, depressive symptomatology (adjusted coefficient 0.076, 95\% $\mathrm{Cl} 0.039$ to $0.113, \mathrm{p}<0.0001$ ) and obesity (adjusted coefficient $0.62,95 \% \mathrm{Cl} 0.038$ to $1.21, \mathrm{p}=0.037$ ) were associated with smaller pain reductions. For procedural outcome, depression (adjusted OR 0.94, 95\% Cl 0.91, $0.97, \mathrm{p}<0.0001$ ) and poorer baseline function (adjusted OR $0.59,95 \% \mathrm{Cl} 0.36,0.96, p=0.034$ ) were associated with failure. Smoking, sleep dysfunction and non-organic signs were associated with negative outcomes in univariate but not multivariate analyses.

Conclusions Identifying treatment responders is a critical endeavor for the viability of procedures in LBP. Patients with greater disease burden, depression and obesity are more likely to fail interventions. Steps to address these should be considered before or concurrent with procedures as considerations dictate.

Trial registration number NCT02329951.

\section{INTRODUCTION}

Low back pain (LBP) is the leading cause of years lost to disability worldwide and in about two-thirds of individual countries. ${ }^{1}$ In a systematic review, Hoy et $a l^{2}$ found the point prevalence to be $18 \%$, annual prevalence to be $38 \%$ and the lifetime prevalence to exceed $40 \%$. There are no reliable treatments for LBP, with many patients receiving injections. In the US fee-for-service Medicare population alone, over 5600000 interventional pain procedures were performed in 2018, with a majority done for LBP. ${ }^{34}$ The three most common procedures are epidural steroid injections (ESIs), facet interventions and sacroiliac joint (SIJ) injections. ${ }^{3}$

There are wide variations in reported success rates for interventional lumbar spine procedures that depend on outcome measures, study design, the specialty of those performing the procedure and the population studied, but in general, geography and non-surgical lumbar procedural volumes are strongly correlated with surgical rates. ${ }^{5}$ This strongly suggests the need for better patient selection, which would improve outcomes, reduce risks, ${ }^{6}$ and save millions of dollars for procedures unlikely to provide long-term benefit.

Personalized medicine involves the utilization of an individual's unique clinical and psychosocial profile to make informed decisions about treatments. Instead of a biomedical approach wherein medical treatments, including procedures, are generically applied in a symptom or diseasebased context (eg, ESI for sciatica), a personalized approach operates in the framework of a biopsychosocial model, focusing on individualized aspects of the pain experience. ${ }^{7}$

The wide variations in outcomes for lumbar spine interventions signal a strong need for studies focused on identifying likely responders, which would favorably alter the risk:benefit ratio. Clinical trials may provide a window into traits associated with treatment response but typically enroll a homogenous population and thus tend to be poorly generalizable. Yet to date, there have been few large-scale studies that sought to determine factors associated with treatment outcome, with most concentrating on clinical signs and symptoms and radiologic pathology, and all focused on a single procedure. 8910

Previously, our group published two articles examining the correlation between specific variables that have not been previously evaluated for their influence on LBP procedural outcomes (hypersensitivity reactions for ESI and non-organic signs for ESI, facet interventions and SIJ injections) as pilot studies during planned interim analyses. ${ }^{611}$ Herein, we present the complete dataset on the effect of over two dozen clinical, psychosocial, technical and condition-specific variables (eg, physical exam signs) on interventional treatment outcomes for 


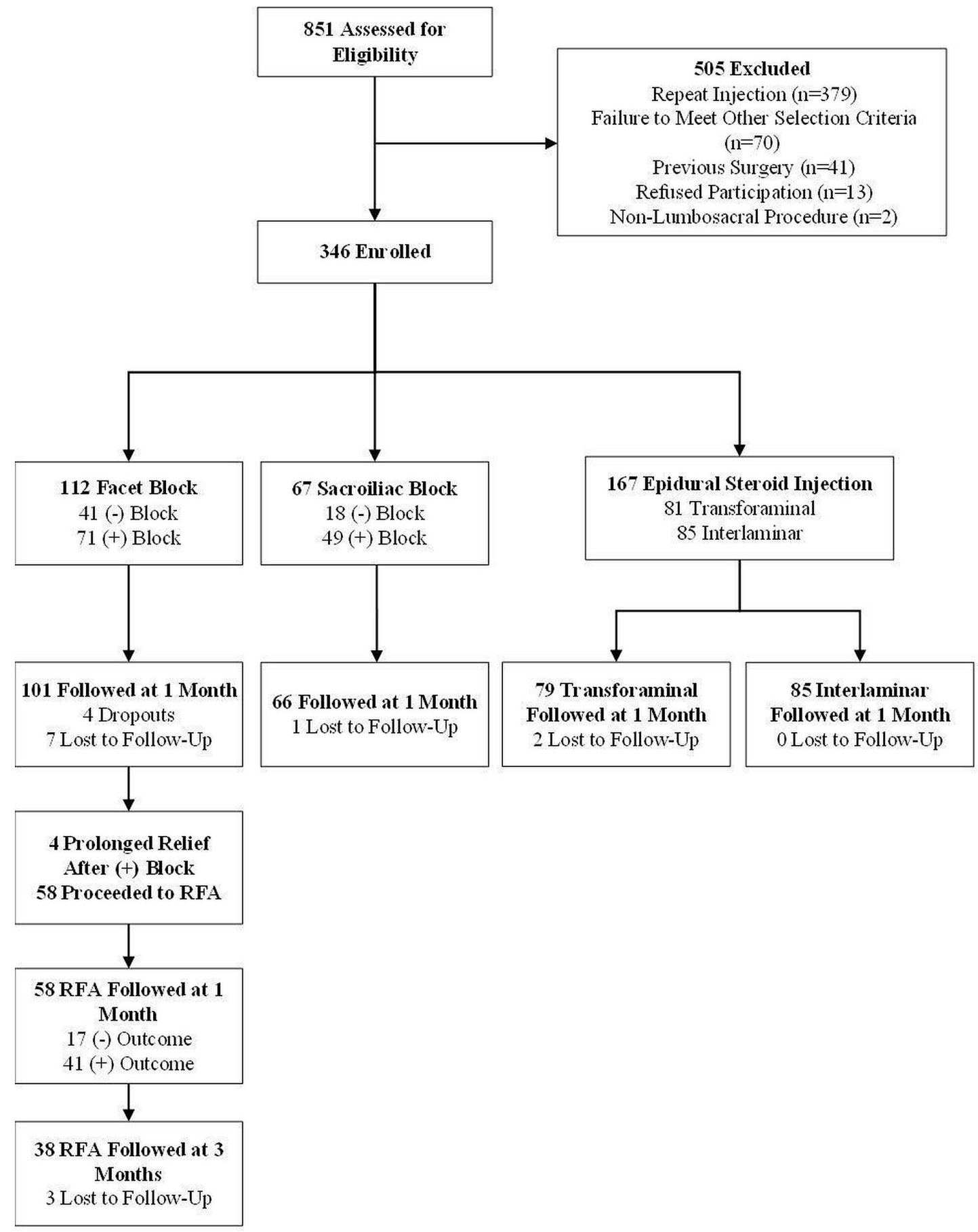

Figure 1 Study flow chart.

Epidural steroid injections include one patient whose injection route was not recorded. RFA, radiofrequency ablation.

chronic LBP. Our objectives were to determine which factors are associated with treatment outcome and quantify the magnitude of effect that could guide pain interventionalists and referring providers in selecting candidates for LBP procedures.

\section{METHODS}

Permission to conduct this multicenter, cohort study was granted by the Institutional Review Boards of Johns Hopkins School of Medicine (IRB00050132), Drexel University, Walter Reed National Military Medical Center, Puget Sound Veterans Affairs (VA) Hospital, Seattle, Washington, Landstuhl Regional Medical Center, Landstuhl, Germany, Naval Medical Center-San Diego and Womack Army Medical Center, Fort Bragg, North Carolina. The study was registered on clinicaltrials.gov on 1 January 2015, with all participants providing informed consent. All enrollments, treatments and follow-up visits took place between 13 January 2015 and 30 May 2021. Detailed descriptions of the procedures have previously been published in manuscripts outlining two planned interim analyses. ${ }^{611}$

\section{Participants and settings}

This study was performed at two civilian, four military and one VA pain treatment centers, four of which had pain medicine training programs. Selection criteria were age $\geq 18$ years; $>6$ 
Table 1 Baseline demographic and clinical characteristics of study subjects

\begin{tabular}{|c|c|c|c|c|c|}
\hline Characteristic & $\begin{array}{l}\text { Facet interventions } \\
(\mathrm{n}=112)\end{array}$ & $\begin{array}{l}\text { Sacroiliac joint } \\
\text { injections } \\
(n=67)\end{array}$ & $\begin{array}{l}\text { Epidural steroid injection } \\
(\mathrm{n}=167)\end{array}$ & $\begin{array}{l}\text { Total cohort } \\
(\mathrm{n}=346)\end{array}$ & P Value* \\
\hline Age, years (mean $\pm S D)$ & $51 \pm 14$ & $53 \pm 16$ & $52 \pm 14$ & $52 \pm 14$ & 0.68 \\
\hline Sex, $n(\%)$ & & & & & 0.091 \\
\hline Female & $53(47.3)$ & $43(64.2)$ & $89(53.3)$ & $161(46.5)$ & \\
\hline Male & $59(52.7)$ & $24(35.8)$ & $78(46.7)$ & $185(53.5)$ & \\
\hline Duration of pain, years $(m e a n \pm S D)$ & $7 \pm 7$ & $5 \pm 6$ & $5 \pm 7$ & $6 \pm 7$ & 0.057 \\
\hline Obesity, n (\%) & $54(48.2)$ & $27(40.3)$ & $74(44.3)$ & $155(44.8)$ & 0.58 \\
\hline Smoking, n (\%) & $24(21.4)$ & $10(14.9)$ & $47(28.1)$ & $81(23.4)$ & 0.081 \\
\hline Bilateral symptoms, n (\%) & $66(60.0)$ & $25(37.9)$ & $59(35.3)$ & $150(43.7)$ & $<0.001$ \\
\hline Inciting event, $n(\%)$ & $41(36.6)$ & $31(46.3)$ & $52(31.1)$ & $124(35.8)$ & 0.091 \\
\hline Opioid use, n (\%) & $21(18.8)$ & $13(19.4)$ & $30(18.0)$ & $64(18.5)$ & 0.96 \\
\hline \multicolumn{6}{|l|}{ Non-opioid medication uset } \\
\hline Any non-opioid & $83(74.1)$ & $47(70.1)$ & $131(78.4)$ & $261(75.4)$ & 0.38 \\
\hline Neuropathic adjuvant & $26(23.2)$ & $14(20.9)$ & $61(36.5)$ & $101(29.2)$ & 0.014 \\
\hline Non-steroidal anti-inflammatory Drug & $54(48.2)$ & $34(50.7)$ & $87(52.1)$ & $175(50.6)$ & 0.82 \\
\hline Muscle relaxant & $22(19.6)$ & $7(10.4)$ & $30(18.0)$ & $59(17.1)$ & 0.26 \\
\hline Other non-opioid analgesic & $10(8.9)$ & $3(4.5)$ & $17(10.2)$ & $30(8.7)$ & 0.37 \\
\hline Tramadol & $16(14.3)$ & $7(10.4)$ & $26(15.6)$ & $49(14.2)$ & 0.6 \\
\hline Active duty, $\mathrm{n}(\%)$ & $27(24.1)$ & $14(20.9)$ & $15(9.6)$ & $57(16.5)$ & 0.013 \\
\hline Disability, worker's compensation or medical board, $n(\%) \ddagger$ & $25(22.9)$ & $16(24.6)$ & $48(29.6)$ & $89(26.5)$ & 0.44 \\
\hline \multicolumn{6}{|l|}{ Coexisting chronic pain condition, $\mathrm{n}(\%) \S$} \\
\hline Any pain condition & $82(73.2)$ & $37(55.2)$ & $91(54.5)$ & $210(60.7)$ & 0.004 \\
\hline Neck pain & $21(18.8)$ & $11(16.4)$ & $25(15.0)$ & $57(16.5)$ & 0.71 \\
\hline Headache & $11(9.8)$ & $6(9.0)$ & $11(6.6)$ & $28(8.1)$ & 0.6 \\
\hline Arthralgia & $55(49.1)$ & $23(34.3)$ & $48(28.7)$ & $126(36.4)$ & 0.002 \\
\hline Neuropathy & $10(8.9)$ & $8(11.9)$ & $17(10.2)$ & $35(10.1)$ & 0.81 \\
\hline Abdominal/pelvic pain & $1(0.9)$ & $4(6.0)$ & $3(1.8)$ & $8(2.3)$ & 0.076 \\
\hline Fibromyalgia & $1(0.9)$ & $2(3.0)$ & $11(6.6)$ & $14(4.0)$ & 0.054 \\
\hline Other chronic pain & $9(8.0)$ & $6(9.0)$ & $12(7.2)$ & $27(7.8)$ & 0.9 \\
\hline Multiple pain conditions & $13(11.6)$ & $9(13.4)$ & $22(13.2)$ & $44(12.7)$ & 0.91 \\
\hline \multicolumn{6}{|l|}{ Coexisting psychiatric condition, $n(\%) q$} \\
\hline Any psychiatric condition & $49(43.8)$ & $27(40.3)$ & $75(44.9)$ & $151(43.6)$ & 0.81 \\
\hline Anxiety & $22(19.6)$ & $14(20.9)$ & $38(22.8)$ & $74(21.4)$ & 0.82 \\
\hline Depression & $25(22.3)$ & $13(19.4)$ & $44(26.3)$ & $82(23.7)$ & 0.48 \\
\hline Post-traumatic stress & $4(3.6)$ & $3(4.5)$ & $7(4.2)$ & $14(4.0)$ & 0.95 \\
\hline Substance abuse history & $7(6.3)$ & $5(7.5)$ & $18(10.9)$ & $30(8.7)$ & 0.37 \\
\hline Other & $5(4.5)$ & $3(4.5)$ & $8(4.8)$ & $16(4.6)$ & 0.99 \\
\hline Multiple & $15(13.4)$ & $5(7.5)$ & $26(15.6)$ & $46(13.3)$ & 0.26 \\
\hline Non-organic signs $(m e a n \pm S D)^{* *}$ & $0.5 \pm 1.0$ & $0.6 \pm 1.2$ & $0.9 \pm 1.3$ & $0.7 \pm 1.2$ & 0.035 \\
\hline Expectations $(m e a n \pm S D)+\dagger$ & $3.6 \pm 1.3$ & $3.9 \pm 1.4$ & $4.0 \pm 1.3$ & $3.9 \pm 1.3$ & 0.047 \\
\hline Athens Insomnia Scale (mean $\pm S D)$ & $9 \pm 5$ & $9 \pm 6$ & $10 \pm 6$ & $9 \pm 5$ & 0.28 \\
\hline Quick Inventory of Depressive Symptomatology (QIDS; mean \pm SD) & $11 \pm 7$ & $11 \pm 9$ & $12 \pm 8$ & $12 \pm 8$ & 0.46 \\
\hline Oswestry Disability Score $(m e a n \pm S D)$ & $36 \pm 15$ & $37 \pm 17$ & $41 \pm 17$ & $39 \pm 16$ & 0.030 \\
\hline \multicolumn{6}{|l|}{ Procedure-related pain (mean $\pm S D$ ) } \\
\hline Skin wheal & $4.7 \pm 2.6$ & $4.7 \pm 2.6$ & $3.9 \pm 2.7$ & $4.3 \pm 2.7$ & 0.019 \\
\hline Procedure & $5.5 \pm 2.7$ & $6.4 \pm 2.7$ & $4.9 \pm 3.0$ & $5.4 \pm 2.9$ & 0.001 \\
\hline Average NRS back pain score $(m e a n \pm S D)$ & $6.0 \pm 1.6$ & $6.2 \pm 1.8$ & $5.9 \pm 2.3$ & $6.0 \pm 2.0$ & 0.46 \\
\hline Worst NRS back pain score (mean \pm SD) & $8.7 \pm 1.3$ & $8.6 \pm 1.4$ & $8.3 \pm 2.5$ & $8.5 \pm 1.9$ & 0.23 \\
\hline Average NRS leg pain score $($ mean $\pm S D)$ & & & $5.9 \pm 2.0$ & & \\
\hline Worst NRS leg pain score $(m e a n \pm S D)$ & & & $8.5 \pm 1.6$ & & \\
\hline
\end{tabular}

*Based on one-way ANOVA for continuous variables and $\chi^{2}$ test for categorical variables.

†Neuropathic adjuvant includes anticonvulsants and antidepressants. Non-steroidal anti-inflammatory drug also includes acetaminophen. Other non-opioid analgesic includes topical agents such as lidocaine and capsaicin.

¥Military equivalent of civilian disability.

$\S$ Denotes active condition (ie, being treated); other includes postsurgical pain, plantar fasciitis, post-stroke pain and pain secondary to systemic disease (eg, lupus and sickle cell anemia).

IDenotes active condition (ie, being treated) except for substance abuse (ongoing recovery); other includes somatic symptom disorder, personality disorder, etc.

**Non-organic signs based on five categories of Waddell signs.

t+Expectations based on 6-point Likert scale ( $1=$ don't expect any relief, $6=$ would only be satisfied with complete pain relief and restoration of function)

ANOVA, analysis of variance; NRS, numerical rating scale.

weeks pain duration; lumbosacral radicular pain or mechanical LBP of presumed facetogenic or sacroiliac joint etiology ${ }^{12}{ }^{13}$; candidate for an ESI, lumbar facet block or SIJ injection and agreement to undergo that injection; and average leg or LBP pain score $\geq 4 / 10$ over the past week for participants with radicular and non-radicular pain, respectively. Excluded from 


\section{Original research}

Table 2 Procedural outcomes

\begin{tabular}{|c|c|c|c|c|c|c|}
\hline \multirow[b]{2}{*}{ Outcome } & \multicolumn{2}{|c|}{ Facet interventions } & \multirow[b]{2}{*}{$\begin{array}{l}\text { Sacroiliac joint } \\
\text { injections } \\
(n=67)\end{array}$} & \multirow[b]{2}{*}{$\begin{array}{l}\text { Epidural steroid } \\
\text { injection } \\
(n=167)\end{array}$} & \multirow[b]{2}{*}{$P$ valuet } & \multirow[b]{2}{*}{$\begin{array}{l}\text { Total cohort } \\
(n=346)\end{array}$} \\
\hline & $\begin{array}{l}\text { All facet } \\
\text { interventions* } \\
(n=112)\end{array}$ & $\begin{array}{l}\text { Radiofrequency } \\
\text { ablation } \\
(n=58)\end{array}$ & & & & \\
\hline Change in average NRS back pain score (mean \pm SD) & $-1.2 \pm 2.4$ & $-2.0 \pm 2.5$ & $-1.8 \pm 2.5$ & $-1.1 \pm 2.4$ & 0.022 & $-1.6 \pm 2.5$ \\
\hline Change in worst NRS back pain score (mean \pm SD) & $-1.6 \pm 2.5$ & $-2.4 \pm 2.7$ & $-2.4 \pm 2.9$ & $-1.7 \pm 3.0$ & 0.14 & $-2.1 \pm 2.9$ \\
\hline Change in average NRS leg pain score (mean \pm SD) & & & & $-1.5 \pm 2.7$ & & \\
\hline Change in worst NRS leg pain score (mean $\pm S D)$ & & & & $-2.4 \pm 3.0$ & & \\
\hline $\begin{array}{l}\text { Change in average NRS primary location pain score } \\
(\text { mean } \pm \text { SD) }\end{array}$ & $-1.2 \pm 2.4$ & $-2.0 \pm 2.5$ & $-1.8 \pm 2.5$ & $-1.5 \pm 2.7$ & 0.50 & $-1.8 \pm 2.6$ \\
\hline $\begin{array}{l}\text { Change in worst NRS primary location pain score } \\
\text { (mean } \pm \text { SD) }\end{array}$ & $-1.6 \pm 2.5$ & $-2.4 \pm 2.7$ & $-2.4 \pm 2.9$ & $-2.4 \pm 3.0$ & 1.00 & $-2.5 \pm 2.9$ \\
\hline Change in Oswestry Disability Score (mean \pm SD) & $-4 \pm 12$ & $-7 \pm 11$ & $-7 \pm 13$ & $-4 \pm 15$ & 0.29 & $-5 \pm 14$ \\
\hline
\end{tabular}

Differences are listed as change from baseline, with negative numbers denoting a decrease in NRS pain score.

*Includes individuals with negative diagnostic medial branch blocks.

tP value is for one-way ANOVA comparison of radiofrequency ablation, sacroiliac joint injections and epidural steroid injections (excludes medial branch blocks).

¥Primary location is back for facet and sacroiliac joint injections, leg for epidural steroid injections.

ANOVA, analysis of variance; NRS, numerical rating scale.

participation were individuals who had received injections within the previous 2 years; with inflammatory arthritis; prior spine surgery for patients undergoing facet blocks and ESI; hypersensitivity reaction to bupivacaine, contrast or corticosteroid; coagulopathy precluding treatment; and pregnancy.

\section{Procedures}

All procedures were performed using fluoroscopic guidance by a board-certified pain medicine physician or trainee under their direct supervision. Procedures were done in accordance with standard protocols and were previously described in detail. ${ }^{6} 111214$
After a $1 \mathrm{~mL}$ skin wheal was created with a 25 -gauge needle using lidocaine 1\%, the soft tissue in the impending needle track(s) was anesthetized using up to $10 \mathrm{~mL}$ of lidocaine $1 \%$, as clinically indicated.

\section{SIJ injections}

SIJ injections were accomplished by inserting 22-gauge needles into the inferior one-third of the joint as previously described. ${ }^{615}$ If the joint capsule was not visualized, the needle was readjusted at the physician's discretion considering the comparable prevalence of intra-articular and extra-articular pathology and

\section{9}

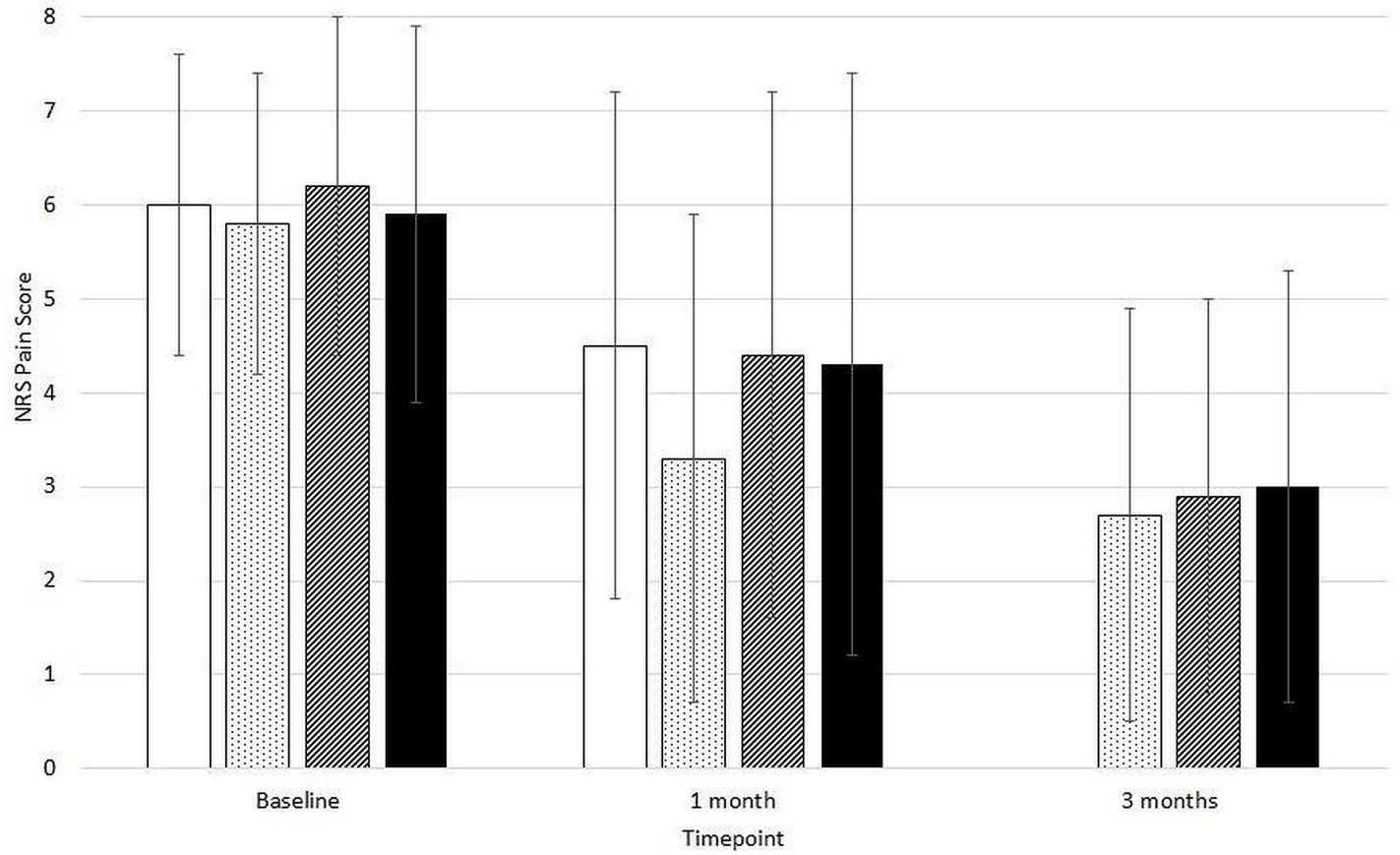

$\square$ Facet Intervention (all) $\square$ Radiofrequency Ablation $\quad$ Sacroiliac Injection Epidural Steroid Injection

Figure 2 Average numerical rating scale pain score at primary pain location (low back for facet and SI, leg for ESI) over time. Note that 'facet intervention (all)' includes both medial branch blocks (including negative blocks) and RFA patients. Patients who did not undergo RFA were not required to follow up at 3 months. ESI, epidural steroid injections; RFA, radiofrequency ablation; 
Original research

Table 3 Demographic and clinical factors associated with treatment outcome

\begin{tabular}{|c|c|c|c|}
\hline Variable & $\begin{array}{l}\text { Procedural failure } \\
(n=193)\end{array}$ & $\begin{array}{l}\text { Procedural } \\
\text { success }(n=139) \dagger\end{array}$ & $P$ value* \\
\hline $\begin{array}{l}\text { Procedure ( } n, \% \text { of } \\
\text { procedure type) }\end{array}$ & & & $0.058 \S$ \\
\hline Facet interventionsł & $68(67.3)$ & $33(32.7)$ & \\
\hline $\begin{array}{l}\text { Radiofrequency } \\
\text { ablation }\end{array}$ & $29 / 58(50)$ & $29 / 58(50)$ & \\
\hline Sacroiliac joint injection & $33(50)$ & $33(50)$ & \\
\hline ESI & $92(55.8)$ & $73(44.2)$ & \\
\hline Transforaminal & $38 / 79(48.1)$ & $41 / 79(51.9)$ & \\
\hline Interlaminar & $53 / 85(62.4)$ & $32 / 85(37.6)$ & \\
\hline Age, years $($ mean $\pm S D)$ & $50 \pm 13$ & $54 \pm 16$ & 0.019 \\
\hline Sex, $n(\%)$ & & & 0.38 \\
\hline Female & $108(60.3)$ & $71(39.7)$ & \\
\hline Male & $85(55.6)$ & $68(44.4)$ & \\
\hline $\begin{array}{l}\text { Duration of pain, years } \\
(\text { mean } \pm \text { SD) }\end{array}$ & $6.7 \pm 7.7$ & $4.7 \pm 5.7$ & 0.01 \\
\hline Obesity, n (\%) & $97(64.2)$ & $54(35.8)$ & 0.039 \\
\hline Bilateral symptoms, n (\%) & $86(61.9)$ & $53(38.1)$ & 0.039 \\
\hline Smoking, n (\%) & $59(75.6)$ & $19(24.4)$ & $<0.001$ \\
\hline Inciting event, n (\%) & $76(63.3)$ & $44(36.7)$ & 0.15 \\
\hline Opioid use, n (\%) & $42(65.6)$ & $22(34.4)$ & 0.18 \\
\hline \multicolumn{4}{|l|}{ Non-opioid use, $\mathrm{n}(\%)$ ๆ } \\
\hline Any adjuvant & $138(55.2)$ & $112(44.8)$ & 0.059 \\
\hline Neuropathic adjuvant & $61(63.5)$ & 35 (36.5) & 0.2 \\
\hline $\begin{array}{l}\text { Non-steroidal anti- } \\
\text { inflammatory drug }\end{array}$ & $87(51.5)$ & $82(48.5)$ & 0.012 \\
\hline Muscle relaxant & $29(49.2)$ & $30(50.8)$ & 0.12 \\
\hline $\begin{array}{l}\text { Other non-opioid } \\
\text { analgesic }\end{array}$ & $15(51.7)$ & $14(48.3)$ & 0.46 \\
\hline Tramadol & $32(66.7)$ & $16(33.3)$ & 0.2 \\
\hline Active duty, n (\%) & $31(57.4)$ & $23(42.6)$ & 0.12 \\
\hline $\begin{array}{l}\text { Disability, worker's } \\
\text { compensation or medical } \\
\text { board, } \mathrm{n}(\%)^{* *}\end{array}$ & $61(70.1)$ & $26(29.9)$ & 0.009 \\
\hline \multicolumn{4}{|c|}{ Coexisting chronic pain conditions, $\mathrm{n}(\%)+\dagger$} \\
\hline Any pain condition & $122(60.4)$ & $80(39.6)$ & 0.3 \\
\hline Neck pain & $32(58.2)$ & $23(41.8)$ & 0.99 \\
\hline Headache & $17(63.0)$ & $10(37.0)$ & 0.6 \\
\hline Arthralgia & $73(59.8)$ & $49(40.2)$ & 0.63 \\
\hline Neuropathy & $19(55.9)$ & $15(44.1)$ & 0.78 \\
\hline Abdominal/pelvic pain & $7(87.5)$ & $1(12.5)$ & 0.088 \\
\hline Fibromyalgia & $8(57.1)$ & $6(42.9)$ & 0.94 \\
\hline Other chronic pain $\ddagger \ddagger$ & $20(80.0)$ & $5(20.0)$ & 0.021 \\
\hline $\begin{array}{l}\text { Multiple pain } \\
\text { conditions }\end{array}$ & $28(65.1)$ & $15(34.9)$ & 0.32 \\
\hline \multicolumn{4}{|c|}{ Coexisting psychiatric condition, $\mathrm{n}(\%) \S \S$} \\
\hline $\begin{array}{l}\text { Any psychiatric } \\
\text { condition }\end{array}$ & $96(66.2)$ & $49(33.8)$ & 0.009 \\
\hline Anxiety & $47(65.3)$ & $25(34.7)$ & 0.16 \\
\hline Depression & $56(70.9)$ & $23(29.1)$ & 0.008 \\
\hline $\begin{array}{l}\text { Posttraumatic stress } \\
\text { disorder }\end{array}$ & $11(78.6)$ & $3(21.4)$ & 0.11 \\
\hline Substance abuse & $22(75.9)$ & $7(24.1)$ & 0.043 \\
\hline Other & $12(75.0)$ & $4(25.0)$ & 0.16 \\
\hline $\begin{array}{l}\text { Multiple psychiatric } \\
\text { conditions }\end{array}$ & $33(71.7)$ & $13(28.3)$ & 0.044 \\
\hline $\begin{array}{l}\text { Non-organic signs (mean, } \\
\text { SD)१ๆ }\end{array}$ & $0.9 \pm 1.3$ & $0.5 \pm 1.0$ & 0.004 \\
\hline
\end{tabular}

Continued
Table 3 Continued

\begin{tabular}{|c|c|c|c|}
\hline Variable & $\begin{array}{l}\text { Procedural failure } \\
(n=193)\end{array}$ & $\begin{array}{l}\text { Procedural } \\
\text { success }(n=139) \dagger\end{array}$ & $P$ value* \\
\hline $\begin{array}{l}\text { Expectations } \\
(\text { mean } \pm S D)^{* * *}\end{array}$ & $3.9 \pm 1.3$ & $3.8 \pm 1.3$ & 0.31 \\
\hline $\begin{array}{l}\text { Athens Insomnia Scale } \\
(\text { mean } \pm \text { SD) }\end{array}$ & $10 \pm 5$ & $8 \pm 5$ & $<0.001$ \\
\hline $\begin{array}{l}\text { Quick Inventory } \\
\text { of Depressive } \\
\text { Symptomatology (QIDS; } \\
\text { mean } \pm \text { SD) }\end{array}$ & $13 \pm 8$ & $9 \pm 7$ & $<0.001$ \\
\hline $\begin{array}{l}\text { Oswestry Disability Score } \\
\text { (mean } \pm \text { SD) }\end{array}$ & $42 \pm 17$ & $35 \pm 15$ & $<0.001$ \\
\hline \multicolumn{4}{|c|}{ Procedure-related pain (mean, SD } \\
\hline Skin wheal & $4.5 \pm 2.7$ & $4.1 \pm 2.7$ & 0.21 \\
\hline Procedure & $5.9 \pm 3.0$ & $4.6 \pm 2.6$ & $<0.001$ \\
\hline $\begin{array}{l}\text { Average baseline back } \\
\text { pain score }(\text { mean } \pm S D)\end{array}$ & $6.3 \pm 2.0$ & $5.6 \pm 2.0$ & 0.002 \\
\hline $\begin{array}{l}\text { Worst baseline back pain } \\
\text { score }(\text { mean } \pm S D)\end{array}$ & $8.8 \pm 1.7$ & $8.1 \pm 2.2$ & 0.001 \\
\hline $\begin{array}{l}\text { Average baseline leg pain } \\
\text { score }(\text { mean } \pm S D)+\dagger \dagger\end{array}$ & $6.2 \pm 2.0$ & $5.4 \pm 1.8$ & 0.006 \\
\hline $\begin{array}{l}\text { Worst baseline leg pain } \\
\text { score }(\text { mean } \pm S D)+\dagger \dagger\end{array}$ & $8.8 \pm 1.6$ & $8.1 \pm 1.6$ & 0.005 \\
\hline
\end{tabular}

*Based on $\mathrm{t}$-test for continuous variables and $\chi^{2}$ test for categorical variables. †Procedural success defined as $\geq 2$-point decrease in baseline average low back or leg pain score at 1 month after sacroiliac joint and epidural steroid injections, respectively, or low back pain score 3 months after radiofrequency ablation (or medical branch blocks for patients with sustained relief) for lumbar facet joint pain, coupled with $>3 / 5$ score on a Likert satisfaction scale.

₹Negative facet interventions include negative RFA outcome and negative facet blocks in patients who did not obtain long-term relief $(n=1)$.

$\S$ Comparison among procedural success for facet RFA at 3 months, SIJ injection at 1 month, and ESI at 1 month.

INeuropathic adjuvants include anticonvulsants and antidepressants. Non-steroidal anti-inflammatory drugs include acetaminophen. Other non-opioid analgesics include topical agents such as lidocaine and capsaicin.

${ }^{* *}$ Military equivalent of civilian disability.

††Denotes active condition (ie, being treated).

$\$ \neq 0$ ther pain conditions include plantar fasciitis, central pain, persistent postsurgical pain and atypical chest pain.

$\S \S$ Denotes active condition (ie, being treated) except for substance abuse. १११Non-organic signs based on five categories of Waddell signs.

***Expectations based on a 6-point Likert scale. t†tLeg pain score only recorded for ESIs.

ESI, epidural steroid injection; RFA, radiofrequency ablation.

evidence for benefit. ${ }^{15}$ Once needle position was deemed sufficient, a $3 \mathrm{~mL}$ solution containing $1 \mathrm{~mL}$ of $40 \mathrm{mg} / \mathrm{mL}$ of depomethylprednisolone and $2 \mathrm{~mL}$ of $0.5 \%$ bupivacaine was injected. A positive diagnostic block was considered $\geq 50 \%$ pain reduction lasting at least 3 hours based on pain diaries with activity logs.

\section{Facet interventions}

Medial branch blocks (MBBs)

The targets for facet interventions were based on historical and physical exam findings, pain referral patterns and radiologic imaging when available. ${ }^{6}{ }^{12} 16$ Twenty-two-gauge needles were inserted between the sacral ala and articular process for the L5 dorsal ramus and just below the junction of the upper transverse process and the superior articular process for L1-4 MBBs with real-time contrast injection. ${ }^{6}{ }^{16}$ At each nerve, $0.5 \mathrm{~mL}$ of $0.5 \%$ bupivacaine was administered. A block was designated as positive if the patient experienced $\geq 50 \%$ pain reduction lasting at least 3 hours. 


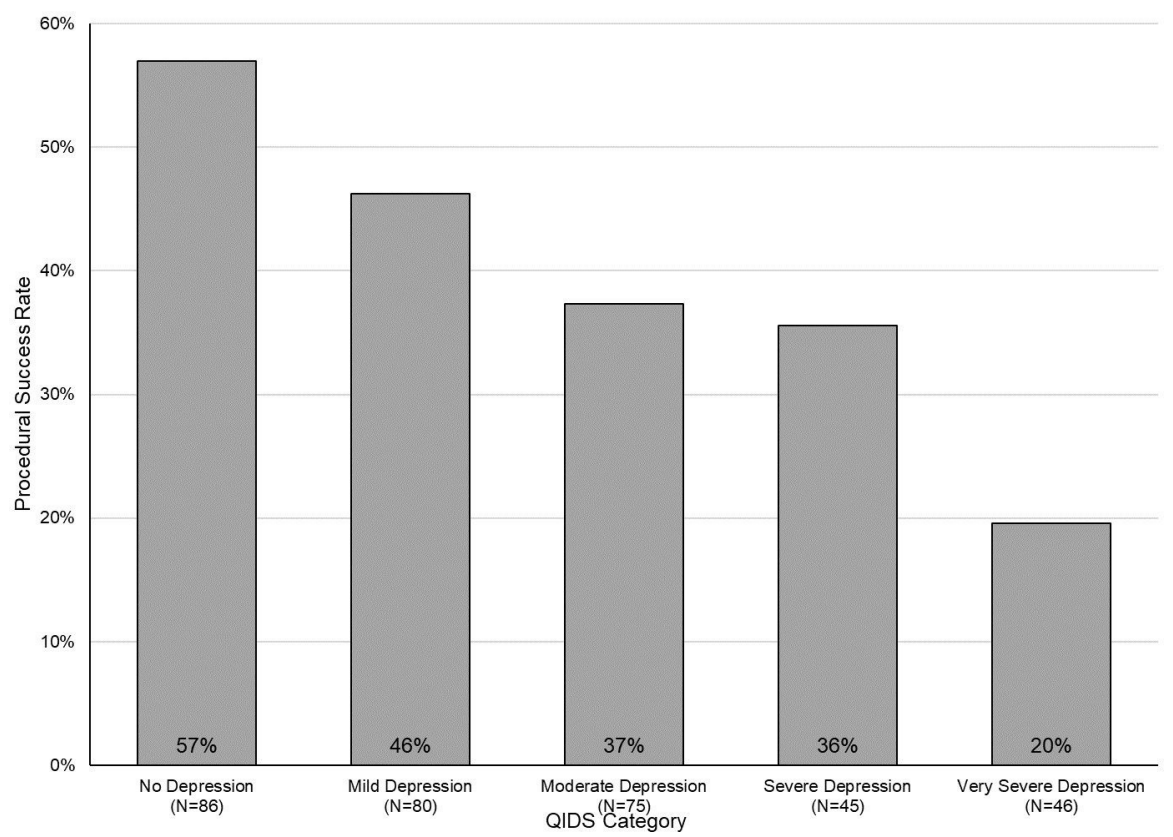

Figure 3 Procedural success by stratified by Quick Inventory of Depressive Symptomatology (QIDS) score. QIDS scores range from 0 to 27 . A score of 5 or lower indicates no depression, 6-10 mild depression, 11-15 moderate depression, 16-20 severe depression and greater than 21 very severe depression.

Lumbar medial branch radiofrequency ablation (RFA)

RFA was performed on participants who had positive diagnostic MBB but failed to experience $>3$ months pain relief in accordance with previous protocols. ${ }^{6}{ }^{6}$ Procedures were performed with 18-gauge or 20-gauge (used in some procedures before January 2019) curved radiofrequency needles with $10 \mathrm{~mm}$ - active tips situated near-parallel to target nerves. ${ }^{6}{ }^{16}$ At each target, electrodes were adjusted to optimize sensory (ideally $<0.6$ volts) stimulation and maximize paraspinal muscle contraction. After appropriate placement, $1 \mathrm{~mL}$ of $2 \%$ lidocaine was injected. Ablation was implemented at $90^{\circ}$ for $135 \mathrm{~s}$ using a radiofrequency generator after which a $0.5 \mathrm{~mL}$ mixture containing $10 \mathrm{mg}$ depomethylprednisolone and saline was administered to prevent neuritis. ${ }^{12}$

\section{Epidural steroid injections}

Our technique for ESI has previously been described for other trials. ${ }^{6}{ }^{11} 17$ A single-level transforaminal ESI (TFESI) was performed in individuals with unilateral pain, while interlaminar ESI (ILESI) were done for bilateral symptoms. Injections were performed at the level thought to be most responsible for pathology unless anatomic considerations dictated otherwise, with real-time contrast confirmation. For TFESI, a $3 \mathrm{~mL}$ of a solution containing $10 \mathrm{mg}$ of dexamethasone, saline and $1 \mathrm{~mL}$ of $0.25 \%$ bupivacaine was administered. ILESI were performed midline in individuals with symmetrical pain or parasagitally when one side was more painful than the other. After proper position was confirmed, a $4 \mathrm{~mL}$ solution containing $40 \mathrm{mg}$ depomethylprednisolone, $1 \mathrm{~mL}$ of $0.25 \%$ bupivacaine and $2 \mathrm{~mL}$ of saline was injected.

\section{Follow-up}

No conterventions between procedures and follow-up visits were permitted, with the exception of negative MBB (small likelihood of long-term benefit) or short-term relief from RFA (>1-month until 3-month follow-up; see online supplemental table 1). Rescue medications provided were non-steroidal anti-inflammatory drugs, tramadol when NSAIDs were contraindicated or ineffective or a $<20 \%$ increase in opioid dose in participants already receiving opioids. The first follow-up visit was 1 month after the ESI, SIJ injection or lumbar medial branch RFA. Participants who experienced a positive binary outcome at their 1-month follow-up visit returned for the final 3-month follow-up visit, while those with a negative outcome exited to receive other treatments. In participants who experienced prolonged benefit (a positive categorical outcome) from the MBB, the initial data collection occurred 1 month after the MBB. In individuals with a positive 1-month facet outcome whose pain returned shortly thereafter and requested treatment, data were recorded before treatment was implemented and carried forward to 3 months.

\section{Data collection}

At all follow-up visits, a disinterested observer collected data via in-person, telemedicine, MyChart (after March 2020) or telephone visits. Baseline data collected included demographic information; general (inciting event, type of injection and laterality) and condition-specific clinical data (eg, exam findings); treatment-specific data (eg, categorization of SIJ injection as intra-articular or extra-articular and type of ESI performed); concomitant pain conditions; number and type of analgesic medications including opioid use stratified by mean morphine equivalents per day (MME); smoking status; coexisting active psychiatric diagnoses; ongoing secondary financial gain (disability, litigation or worker's compensation case); average and worst back and leg (for ESI patients) pain score on a 0-10 numerical rating scale (NRS) over the past week; number and type of non-organic (Waddell) signs ${ }^{6}$; expectations; Oswestry Disability Index version 2 (ODI v.2) score; Athens Insomnia Scale (AIS) to measure sleep quality; and Quick Inventory of Depressive Symptomatology (QIDS) to measure mood. For ODI, AIS and QIDS, higher scores represent greater dysfunction.

Preprocedure expectations were measured on a 6-point Likert scale based on the degree of improvement the participant would be satisfied with (1=not expecting pain relief, but 
Table 4 Procedure-specific clinical variables associated with treatment outcome

\begin{tabular}{|c|c|c|c|}
\hline Clinical variable & $\begin{array}{l}\text { Procedural } \\
\text { failure }(n=193)\end{array}$ & $\begin{array}{l}\text { Procedural } \\
\text { success } \\
(n=139)^{*}\end{array}$ & $P$ value \\
\hline Epidural steroid injections & 90 & 73 & \\
\hline \multicolumn{4}{|l|}{ Physical exam } \\
\hline Straight leg raising test (-) & $39(43)$ & $32(44)$ & 0.95 \\
\hline Straight leg raising test (+) & $51(57)$ & $41(56)$ & \\
\hline \multicolumn{4}{|l|}{ Pathology } \\
\hline Herniated disc & $42(48)$ & $32(45)$ & 0.13 \\
\hline Spinal stenosis & $31(35)$ & $28(39)$ & \\
\hline $\begin{array}{l}\text { Herniated disc and spinal } \\
\text { stenosis }\end{array}$ & $4(5)$ & $8(11)$ & \\
\hline $\begin{array}{l}\text { Spondylosis without } \\
\text { compression }\end{array}$ & $11(13)$ & $3(4)$ & \\
\hline Facet interventions & 68 & 33 & \\
\hline $\begin{array}{l}<50 \% \text { pain relief from } \\
\text { diagnostic block }\end{array}$ & $36(53)$ & $1(3)$ & - \\
\hline $\begin{array}{l}50 \%-79 \% \text { pain relief from } \\
\text { diagnostic block }\end{array}$ & $23(34)$ & $15(45)$ & $<0.001^{*}$ \\
\hline $\begin{array}{l}\geq 80 \% \text { pain relief from } \\
\text { diagnostic block }\end{array}$ & $9(13)$ & $17(52)$ & $<0.001 \dagger$ \\
\hline Sacroiliac joint injection & 33 & 33 & \\
\hline \multicolumn{4}{|l|}{ Physical exam } \\
\hline Gaenslen's test (-) & $7(21)$ & $10(30)$ & 0.4 \\
\hline Gaenslen's test $(+)$ & $26(79)$ & $23(70)$ & \\
\hline Patrick's test (-) & $9(27)$ & $15(45)$ & 0.12 \\
\hline Patrick's test (+) & $24(73)$ & $18(55)$ & \\
\hline $\begin{array}{l}\text { Positive Patrick's \& Gaenslen's } \\
\text { tests }\end{array}$ & $22(67)$ & $15(45)$ & 0.083 \\
\hline \multicolumn{4}{|l|}{ Prior surgery } \\
\hline Prior back surgery & $7(21)$ & $4(13)$ & 0.35 \\
\hline No prior back surgery & $26(79)$ & $28(88)$ & \\
\hline $\begin{array}{l}<50 \% \text { pain relief from } \\
\text { diagnostic block }\end{array}$ & $15(45)$ & $3(9)$ & - \\
\hline $\begin{array}{l}50 \%-79 \% \text { pain relief from } \\
\text { diagnostic block }\end{array}$ & $9(27)$ & $17(52)$ & $<0.001^{*}$ \\
\hline $\begin{array}{l}\geq 80 \% \text { pain relief from } \\
\text { diagnostic block }\end{array}$ & $9(27)$ & $13(39)$ & $0.30 \dagger$ \\
\hline
\end{tabular}

${ }^{*}$ Comparison of procedural failure versus success based on $\geq 50 \%$ vs. $<50 \%$ pain relief threshold as definition of positive block.

†Comparison of procedural failure versus success based on $\geq 80 \%$ vs. $<80 \%$ pain relief threshold as definition of positive block.

trying anyway; $2=$ any improvement; $3=30 \%-49 \%$ improvement; $4=50 \%-74 \%$ improvement; $5=75 \%-99 \%$ improvement; $6=$ only complete resolution of all symptoms). Conditionspecific provocative tests evaluated included straight leg raising test for individuals receiving ESI and Gaenslen's and Patrick's tests before SIJ injections. At the time of the procedure (MBB but not RFA), a subcutaneous skin wheal was created by a $1 \mathrm{~mL}$ lidocaine injection using a 25 -gauge needle, immediately after which the pain intensity of the injection was assessed via a $0-10$ verbal rating scale. After the procedure, procedure-related pain was measured using the same verbal rating scale. These data were collected because all have been associated with LBP procedure outcomes. ${ }^{17-20}$

\section{Outcome measures}

The primary outcome measure was change in average LBP or leg pain (for ESI) score over the past week. The primary endpoint was 1-month postinjection for ESI and SIJ injections, and 3 months after lumbar medial branch RFA (or MBB in those with prolonged pain relief). Specific scenarios for individuals with a negative $\mathrm{MBB}$, prolonged relief after their $\mathrm{MBB}$, or relief after RFA lasting 1 but not 3 months are noted in online supplemental table 1 . Hereafter, average NRS-PI (pain intensity) refers to the primary outcome measure at the primary endpoint.

A positive categorical response for procedure success was predesignated as a two-point or greater decrease in the NRS-PI not attributable to starting a rescue medication in conjunction with a score of greater than 3 on a 5 -point Likert scale measuring procedural satisfaction ( $1=$ very unsatisfied, $2=$ unsatisfied, $3=$ neither satisfied nor unsatisfied, $4=$ satisfied and $5=$ very satisfied). ${ }^{21}$ At each follow-up, along with average pain scores, secondary outcome measures recorded included worst pain scores over the past week, along with ODI 2.1, satisfaction score, and medication reduction, defined per previous studies to be $>20 \%$ decrease in opioid use or cessation of a non-opioid analgesic. $^{111516}$

\section{Statistical analysis}

Sample size calculation and power analysis were performed for the binary categorical outcome because of differences in effect sizes for the three procedures. ${ }^{22}$ Assuming a procedural success rate of $45 \%$ and a SD of 2.5 points in NRS-PI in both groups, we estimated a sample size of 311 patients would be $94 \%$ powered to detect a 1.0-point minimal clinically important difference between responders and non-responders in NRS-PI at the primary endpoint, at a significance level of $0.05 .^{23}$ Accounting for dropouts, we planned to enroll 346 participants.

All statistical analyses were performed in Stata/IC V.16.1 (StataCorp, USA). For continuous outcomes, group means and SD were reported, and analysis of variance (ANOVA) was used to compare the three procedural groups (facets, SIJ and ESI). For categorical outcomes, percentages were reported, and $\chi^{2}$ tests were used to compare the three procedural groups. A p value less than alpha of 0.05 was considered statistically significant for comparisons between two groups. For comparisons among three or more groups, a Bonferroni-corrected alpha of $0.05 / n$, where $n$ is the number of between-group comparisons, was used.

The primary outcome, average NRS-PI, was analyzed with the last observation carried forward (including from baseline for those who missed 1 month visits) in the case of missing data. Within each treatment group, average baseline NRS and average NRS-PI were compared using paired t-tests. Changes in average NRS-PI, worst NRS-PI and ODI were compared using three-way ANOVA.

For the categorical response of treatment outcome, demographic, patient-specific and procedure-specific variables were reported as above and compared between the procedural failure and success groups. ANOVA was used for continuous variables, and $\chi^{2}$ tests were used for categorical variables.

To identify potential predictors associated with positive outcomes, we performed two multivariate analyses. Change in average NRS-PI was modeled as a function of 10 candidate variables selected based on a combination of our univariate analyses, clinical knowledge and review of relevant literature. Each candidate variable was modeled individually using a simple linear regression model (unadjusted), then with a multivariable model using all 10 variables (full model). ${ }^{23}$ To identify the variables most likely to impact outcome, the multivariable linear regression model was simplified using a backward stepwise approach. A two-sided $\mathrm{p}$ value less than 0.05 was considered statistically 
significant. A non-parametric bootstrap method using 100 resamples was used to internally validate the proposed regression model. Similarly, for the second exploratory analysis, treatment outcome was modeled using logistic regression, with the same 10 candidate variables identified above. ORs are reported for the unadjusted analyses, full model and a simplified model developed using a backward stepwise approach. Again, a two-sided p value less than 0.05 was considered statistically significant and a non-parametric bootstrap method using 100 resamples was used for internal validation.

\section{RESULTS}

A total of 346 patients were enrolled: 112 underwent lumbar facet blocks, 67 received SIJ injections and 167 underwent lumbar ESI (81 transforaminal, 85 interlaminar). Of these patients, 331 were followed up at 1 month (figure 1). For facet block patients who proceeded to RFA, 58 were followed up at 1 month, and 38 were followed 3 months after RFA.

\section{Baseline characteristics}

Table 1 shows baseline demographic and clinical characteristics. Participants in the facet group were significantly more likely to have bilateral pain, have a coexisting chronic pain condition and reported lower expectations for pain relief. The ESI group was significantly more likely to be receiving neuropathic adjuvants, less likely to be in the military, demonstrated more non-organic signs, had slightly higher ODI scores and reported lower mean procedure-related pain during both the skin wheal and the procedure itself. There were no significant differences in other variables.

\section{Procedural outcomes}

For all procedures (facet blocks, RFA, SIJ injections and ESI), there was a significant decrease in mean pain NRS pain score at the primary pain location from baseline to the primary endpoint (1 month for facet blocks, SIJ injections and ESI; 3 months for RFA) (figure 2). Mean decrease in average NRS pain score was $1.2 \pm 2.4$ (95\% CI 0.8 to $1.7, \mathrm{p}<0.0001$ ) for all facet interventions (includes negative MBB), $2.0 \pm 2.5$ (95\% CI 1.3 to 2.6, $\mathrm{p}<0.0001)$ for RFA, $1.8 \pm 2.5$ (95\% CI 1.2 to $2.4, \mathrm{p}<0.0001)$ for SIJ injections and $1.5 \pm 2.7$ (95\% CI 1.1 to $1.9, \mathrm{p}<0.0001)$ for ESI. Among the 139 patients with a successful outcome, the mean reductions in NRS-PI for facet interventions, SIJ injections and ESI were $4.0 \pm 1.5,3.6 \pm 2.0,3.6 \pm 1.9$, respectively. Procedural outcomes are summarized in table 2 . There were no significant differences among the RFA, SIJ injections and ESI groups for change in average or worst back or leg pain scores or ODI.

\section{Factors associated with categorical treatment outcome}

A comparison of clinical and demographic characteristics stratified by procedural outcome is shown in table 3 . In univariate analyses, patients having successful treatment outcomes were significantly more likely to be older, have shorter duration of pain, be receiving non-steroidal anti-inflammatory drugs and have other chronic pain conditions (eg, postsurgical pain and pain secondary to systemic disease). They were significantly less likely to be obese, smoke, have bilateral pain, be on disability/ worker's compensation/medical board, and have any or multiple coexisting psychiatric conditions, most prominently depression (figure 3). As a group, patients with treatment success had fewer non-organic signs, lower AIS, QIDS and ODI scores, reported less procedure-related pain and had lower baseline average and worst pain scores compared with patients who failed treatment.
Procedure type, sex, presence of an inciting event, opioid use, coexisting chronic pain conditions, patient expectations for pain relief and pain due to skin wheal were not associated with outcome.

Table 4 shows procedure-specific characteristics for treatment outcome. For facet interventions, both $\geq 50 \%$ and $\geq 80 \%$ pain relief from diagnostic block were significantly associated with procedural success. For SIJ injections, $\geq 50 \%-79 \%$ pain relief immediately following the procedure was associated with treatment success, while $\geq 80 \%$ relief was actually associated with a lower success rate (52\% vs 39\%). Physical exam findings and history of prior surgery were not significantly different between SIJ injection successes and failures. Similarly, physical exam findings and spinal pathology were not significantly different between patients with procedural success versus failure in the ESI group.

\section{Multivariate regression models}

Based on the previous results, prior evidence and clinical knowledge, the following variables were selected for multivariate analyses: age, duration of pain, obesity, smoking status, opioid use, sleep disturbance (AIS), depression (QIDS), non-organic signs (Waddell), ODI and baseline NRS-PI. Coefficients for linear regressions of change for NRS-PI are shown in table 5 as a function of unadjusted (univariate) and adjusted (full and simplified models) covariates. Procedure type was not chosen as a covariate since it was not associated with outcome. The full model contained all selected variables, while the simplified model was generated by eliminating variables using a backward stepwise approach. In the simplified model, obesity, QIDS and baseline NRS pain score were selected as the final variables. Obesity and higher QIDS score (greater depression) were negatively associated with change in average pain score (ie, smaller decrease in pain score), while higher baseline pain score was positively associated with change in pain (i.e. greater chance to decrease NRS pain score). The presence of obesity was associated with a 0.62 -point increase in change in NRS pain score (ie, worse pain relief, 95\% CI 0.038 to $1.21, \mathrm{p}=0.037$; online supplemental figure 3). Every 1-point increase in QIDS was associated with a 0.076-point decrease in pain score improvement (95\% CI 0.039 to $0.113, \mathrm{p}<0.0001)$, while every 1 -point increase in baseline NRS pain score was associated with a 0.32 -point improvement in NRS pain score (95\% CI 0.16 to $0.48, \mathrm{p}<0.0001)$.

Unadjusted and adjusted ORs for logistic regression analyses of procedural outcome as a function of the same ten selected variables are shown in table 6 . As with linear regression, the full model contained all variables, while the simplified model was generated by eliminating variables using a backward stepwise approach. In the simplified model, baseline QIDS and ODI were selected as final variables. In this model, every 1-point increase in QIDS was associated with a 0.94 times decreased odds of procedural success (95\% CI 0.91 to $0.97, \mathrm{p}<0.0001$ ), while every 1-point increase in ODI was associated with a 0.59 times decreased odds of success $(95 \%$ CI 0.36 to $0.96, p=0.034)$. In other words, there was a $6 \%$ decrease in the odds of procedural success with a 1-point increase in baseline QIDS score, and there was a $41 \%$ decrease in the odds of procedural success with each 1-point increase in baseline ODI score. For NRS-PI, unlike for pain score reduction, a higher baseline pain score was associated with decreased likelihood of achieving a positive outcome. Figure 3 shows the decline in procedural success rate with worsening depression, while figure 4 shows a similar decline in procedural success rate with worsening disability. 
Original research

Table 5 Factors associated with primary outcome (change in average NRS primary location pain score) for therapeutic LBP procedures

\begin{tabular}{|c|c|c|c|c|c|c|}
\hline Variable & Unadjusted coefficient $(95 \% \mathrm{Cl})$ & $P$ value & $\begin{array}{l}\text { Adjusted coefficient, full model } \\
(95 \% \mathrm{Cl})\end{array}$ & $P$ value & $\begin{array}{l}\text { Adjusted coefficient, } \\
\text { simplified model }(95 \% \mathrm{CI})\end{array}$ & $P$ value \\
\hline Age, years & $-0.030(-0.051$ to -0.009$)$ & 0.006 & $-0.021(-0.043$ to 0.00047$)$ & 0.055 & & \\
\hline Duration of pain, years & $0.034(-0.010$ to 0.079$)$ & 0.13 & $0.026(-0.018$ to 0.070$)$ & 0.25 & & \\
\hline Obesity & $0.53(-0.07$ to 1.13$)$ & 0.084 & $0.56(-0.034$ to 1.15$)$ & 0.065 & $0.62(0.038$ to 1.21$)$ & 0.037 \\
\hline Smoking & $0.64(-0.091$ to 1.37$)$ & 0.086 & $0.45(-0.29$ to 1.19$)$ & 0.24 & & \\
\hline Any daily opioid use & $0.32(-0.29$ to 0.93$)$ & 0.30 & $0.39(-0.24$ to 1.02$)$ & 0.22 & & \\
\hline $\begin{array}{l}\text { Athens Insomnia Scale } \\
(m e a n \pm S D)\end{array}$ & $0.067(0.013$ to 0.12$)$ & 0.015 & $0.0072(-0.071$ to 0.086$)$ & 0.86 & & \\
\hline $\begin{array}{l}\text { Quick Inventory of Depressive } \\
\text { Symptomatology (mean } \pm \text { SD) }\end{array}$ & $0.060(0.023$ to 0.098$)$ & 0.002 & $0.044(-0.012$ to 0.099$)$ & 0.12 & $0.076(0.039$ to 0.113$)$ & $<0.0001$ \\
\hline Non-organic signs, number & $0.19(-0.059$ to 0.45$)$ & 0.131 & $0.06(-0.23$ to 0.35$)$ & 0.68 & & \\
\hline $\begin{array}{l}\text { Oswestry Disability Score } \\
\text { (mean } \pm \text { SD) }\end{array}$ & $0.014(-0.0040$ to 0.032$)$ & 0.13 & $0.0016(-0.0074$ to 0.039$)$ & 0.18 & & \\
\hline $\begin{array}{l}\text { Average baseline NRS primary } \\
\text { pain location score }\end{array}$ & $-0.23(-0.39$ to -0.072$)$ & 0.004 & $-0.39(-0.57$ to -0.20$)$ & $<0.0001$ & $-0.32(-0.48$ to -0.16$)$ & $<0.0001$ \\
\hline
\end{tabular}

\section{Complications}

There were 10 complications in the ESI group (6.0\%), which included inadvertent dural puncture $(n=3)$ with one spinal headache, temporary worsening pain $(n=4)$ or new neurologic symptoms $(n=2)$, and an accidental intradiscal injection. In the facet intervention group, there were nine complications $(8.0 \%)$ that included a panic attack, a pseudoseizure, a patient who reported insomnia for several weeks, two vasovagal episodes, two cases of neuritis and two cases of new temporary pain (leg cramp) or neurologic symptoms (shooting pain in leg). In the SIJ injection group, there was one case of worsening pain and one vasovagal episode for a complication rate of $3.0 \%$.

\section{DISCUSSION}

Personalized medicine represents one of the fastest growing areas in pain medicine and may be particularly relevant for interventional procedures, which are characterized by high risks, costs and, in some cases, rewards. ${ }^{24}$ This study is the largest to date examining the effect of dozens of factors on interventional LBP outcomes and examined several factors never evaluated for their effects on outcomes (eg, physical exam signs for SI joint outcomes, expectations, coexisting pain conditions and nonopioid medication usage).

\section{Comparison with other studies}

Some of our findings have been reported for other pain conditions. The negative effects of smoking, disease burden (duration of pain, higher pain scores and greater disability), secondary gain, sleep dysfunction and psychiatric comorbidities have been shown to adversely affect LBP outcomes in both interventional and noninterventional studies. ${ }^{12} 1425-28$ Our results demonstrating how varying degrees of depression and disability linearly correlate, in a dramatic fashion, with outcome, have not previously reported for procedural interventions and suggest that even modest improvement in poorly controlled depression and severe disability may yield fruitful results. Yet, other findings were unexpected. We hypothesized that more chronic pain conditions, which may indicate central sensitization or somatization, would foretell treatment failure, as has been shown in other studies. ${ }^{29}{ }^{30}$ However, we found no significant association between most chronic pain conditions and interventional treatment outcomes, which may reflect differences in treatment (interventional vs conservative), methodology and

\begin{tabular}{|c|c|c|c|c|c|c|}
\hline Variable & Unadjusted OR $(95 \% \mathrm{Cl})^{*}$ & $P$ value & $\begin{array}{l}\text { Adjusted OR, full model }(95 \% \\
\text { Cl)* }\end{array}$ & $P$ value & $\begin{array}{l}\text { Adjusted OR, revised } \\
\text { model }(95 \% \mathrm{Cl})^{*}\end{array}$ & $P$ value \\
\hline Age, years & $1.02(1.00$ to 1.03$)$ & 0.039 & $1.02(1.00$ to 1.04$)$ & 0.024 & & \\
\hline Duration of pain, years & $0.96(0.93$ to 1.00$)$ & 0.051 & 0.96 (0.93 to 1.00$)$ & 0.072 & & \\
\hline Obesity & 0.58 (0.36 to 0.92$)$ & 0.022 & 0.62 (0.37 to 1.03$)$ & 0.066 & & \\
\hline Smoking & $0.41(0.23$ to 0.74$)$ & 0.003 & $0.64(0.33$ to 1.24$)$ & 0.19 & & \\
\hline Any daily opioid use & 0.68 (0.42 to 1.12$)$ & 0.13 & 0.75 (0.42 to 1.35$)$ & 0.34 & & \\
\hline Athens Insomnia Scale $($ mean $\pm S D)$ & 0.93 (0.89 to 0.97$)$ & 0.002 & 1.01 (0.95 to 1.09$)$ & 0.66 & & \\
\hline $\begin{array}{l}\text { Quick Inventory of Depressive } \\
\text { Symptomatology (mean } \pm \text { SD) }\end{array}$ & 0.94 (0.91 to 0.97$)$ & $<0.0001$ & 0.96 (0.91 to 1.00$)$ & 0.077 & 0.94 (0.91 to 0.97 ) & $<0.0001$ \\
\hline Non-organic signs, number & $0.73(0.59$ to 0.90$)$ & 0.004 & 0.94 (0.72 to 1.22$)$ & 0.63 & & \\
\hline Oswestry Disability Score (mean \pm SD) & 0.97 (0.96 to 0.99 ) & $<0.0001$ & $0.99(0.97$ to 1.01$)$ & 0.31 & 0.59 (0.36 to 0.96$)$ & 0.034 \\
\hline $\begin{array}{l}\text { Average baseline NRS primary pain } \\
\text { location score }{ }^{-}\end{array}$ & 0.86 (0.76 to 0.98$)$ & 0.019 & 0.94 (0.80 to 1.10$)$ & 0.41 & & \\
\hline
\end{tabular}

*ORs are reported as likelihood of procedural success with presence (for categorical variables) or each single unit increase (for continuous variables) in the specified variable, with ORs greater than one being associated with increased likelihood of procedural success.

tPrimary location is back for facet and SIJ injections, leg for ESI.

ESI, epidural steroid injection. 


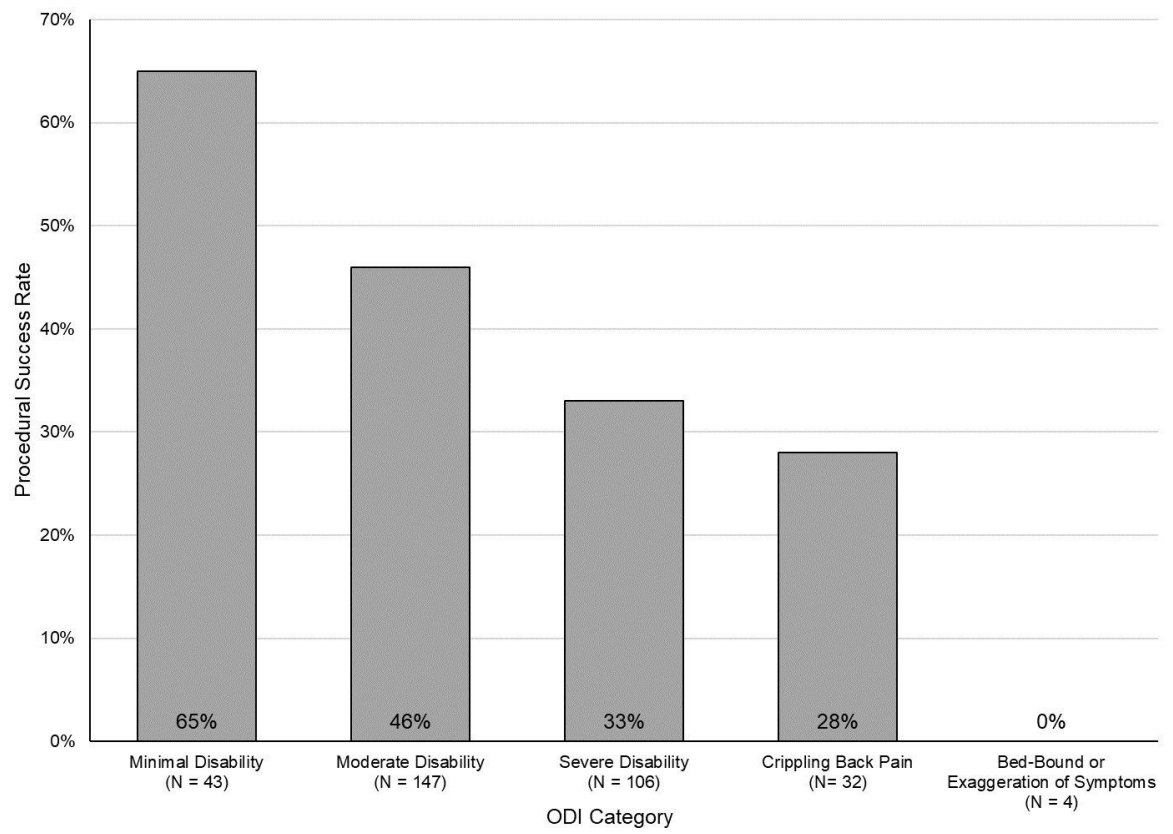

Figure 4 Procedural success by Oswestry Disability Index (ODI) score. ODI scores range from 0\% (no disability) to 100\% (maximum possible disability). A score of $20 \%$ or lower indicates minimal disability, $21 \%-40 \%$ moderate disability, $41 \%-60 \%$ severe disability, $61 \%-80 \%$ crippling back pain and $81 \%$ or greater indicates a patient is either bed-bound or has exaggeration of symptoms.

our relatively short-term follow-up. Unlike other studies, we found no meaningful relationship between preprocedure expectations and treatment results. Whereas most studies have found a positive relationship between expectations for benefit and pain treatment outcomes, ${ }^{31}$ our categorical outcome was tied to procedural satisfaction, and studies have also found that unrealistic outcomes correlate with dissatisfaction with treatment. ${ }^{32}$ We also found no association between the present of an inciting event and procedure success, which is consistent with a study that examined the association in individuals with sciatica but divergent with the results of a study that found a positive correlation in individuals who underwent lumbar medial branch RFA. ${ }^{33}$ In contrast with a prospective study that found pain rating in response to a subcutaneous skin wheal (standardized pain stimulus) predicted less pain relief with ESI, we found no correlation between treatment outcome and pain score after a skin wheal. ${ }^{17}$ However, we did find that a higher procedure-related pain score was associated with poorer treatment outcome. This is surprising since procedure-related stimuli cannot be standardized, and most but not all prior studies on quantitative sensory testing found an inverse relationship between pain thresholds and interventional treatment outcomes. ${ }^{35-37}$ Although a standardized pain stimulus could theoretically be used when making treatment decisions, procedure-related pain cannot.

Our study also examined exam findings and immediate (ie, diagnostic) block results on intermediate-term outcomes. For ESI, unlike previous studies, we found that neither straight leg raising test nor herniated disc were associated with a positive outcome, though individuals with non-compressive etiologies (eg, spondylosis) fared worse. ${ }^{81418}$ For SIJ injections, no significant associations were noted for two of the most common provocative tests. Prior studies have found that while no single test is specific for identifying a painful SIJ, a battery of three or more positive provocative tests is associated with a positive response to diagnostic injections, and a positive diagnostic injection was present in $91 \%$ of those who experienced a positive outcome at 1 month in our study. ${ }^{389}$ Similar to recommendations in lumbar facet guidelines, we found that a higher degree of pain relief was more likely to lead to a positive outcome after
RFA but that setting the threshold for a positive diagnostic block at $80 \%$ would have led to a substantial proportion of patients who benefitted from treatment being denied access. ${ }^{12}$ For SIJ pain, unlike those who obtained between $50 \%$ and $79 \%$ pain relief, experiencing greater $\geq 80 \%$ immediate pain relief did not statistically separate on 1 month outcome from having $<80 \%$ relief, which suggests that many of these patients may have been placebo responders.

\section{Limitations}

There are several limitations that warrant consideration. First, because this study was designed to determine factors associated with treatment outcome in a practice setting, our inclusion criteria were less stringent than typically employed in a clinical trial. Second, there is inter-rater reliability variability regarding some of the factors we examined such as radiologic findings, physical exam findings and even what constitutes an active medical or psychiatric problem. Third, we combined three different LBP treatments that address different etiologies, and it is therefore possible that our study may have been underpowered to detect factors that may predict treatment outcome for one but not the other procedures. These conditions also contain different pathophysiologies (eg, neuropathic vs non-neuropathic pain) and hence may be associated with different outcome predictors; however, there is extensive overlaps in outcome predictors for nearly all chronic pain conditions, ${ }^{14252839}$ and few cohort studies examining LBP outcome predictors differentiate between etiologies. Last, all of the participating institutions in this study were teaching hospitals, which may limit the generalization to private practice settings.

\section{FUTURE RESEARCH AND CONCLUSIONS}

The continued rise in interventions for LBP, with no appreciable effect on disability rates, has led to increased scrutiny from payers and regulators and a push towards precision medicine, tailoring treatment to individual patient characteristics rather than symptoms and pathology. ${ }^{13}$ When employed indiscriminately, LBP interventions are not cost-effective, ${ }^{40}$ but refining selection based on our 
findings and those in large registries and clinical trials can favorably alter risk:benefit ratios and cost-effectiveness. However, physicians should consider that evidence-based medicine involves taking into consideration not just data from research studies but an individual patient's unique circumstances and goals; hence, values-based medicine should be viewed as complementary rather than mutually exclusive with evidence-based medicine. ${ }^{41}$ Given our findings, interventions that might be considered before or concurrent with LBP interventions including psychotherapy or psychotherapeutics in individuals with depression, functional restoration in obese patients and those with high baseline disability, and referrals for smoking cessation in individuals amenable to quitting.

\section{Twitter Tina L Doshi @dr_tinadoshi}

Contributors Study design: SPC. Drafting manuscript: SPC, TLD and CK. IRB submission and progress reports, enrolling and treating patients and critical evaluation of manuscript: all authors. Funding: PFP and SPC. Guarantor: SPC

Funding Funded in part by a grant from MIRROR, Uniformed Services University of the Health Sciences, US Department of Defense, grant \# HU00011920011.

Disclaimer The views expressed in this manuscript are those of the authors and do not reflect the official policy of the Departments of Army and Navy, Department of Defense or US Government. The identification of specific products or scientific instrumentation is considered an integral part of the scientific endeavor and does not constitute endorsement or implied endorsement on the part of the authors, DoD or any component agency. The views expressed in this article those of the authors and do not reflect the official policy of the Departments of Army and Navy, Department of Defense or US Government.

Competing interests SPC: consulting work in past 3 years for Avanos, SPR Therapeutics, Persica, Scilex and Relieviate.

Patient consent for publication Not applicable.

Provenance and peer review Not commissioned; externally peer reviewed.

Data availability statement Data are available on reasonable request. Deidentified data will be available on reasonable request.

\section{ORCID iDs}

Steven P Cohen http://orcid.org/0000-0001-5928-2127

Tina L Doshi http://orcid.org/0000-0001-5011-3298

\section{REFERENCES}

1 GBD 2017 Disease and Injury Incidence and Prevalence Collaborators. Global, regional, and national incidence, prevalence, and years lived with disability for 354 diseases and injuries for 195 countries and territories, 1990-2017: a systematic analysis for the global burden of disease study 2017. Lancet 2018:392:1789-858.

2 Hoy D, Bain C, Williams G, et al. A systematic review of the global prevalence of low back pain. Arthritis Rheum 2012;64:2028-37.

3 Manchikanti L, Sanapati MR, Pampati V, et al. Update on reversal and decline of growth of utilization of interventional techniques in managing chronic pain in the Medicare population from 2000 to 2018. Pain Physician 2019;22:521-36.

4 Manchikanti L, Soin A, Mann DP, et al. Comparative analysis of utilization of epidural procedures in managing chronic pain in the Medicare population: pre and post Affordable care act. Spine 2019;44:220-32.

5 Friedly J, Chan L, Deyo R. Geographic variation in epidural steroid injection use in Medicare patients. J Bone Joint Surg Am 2008;90:1730-7.

6 Cohen SP, Doshi TL, Kurihara C, et al. Waddell (nonorganic) signs and their association with interventional treatment outcomes for low back pain. Anesth Analg 2021;132:639-51.

7 Roth RS, Geisser ME, Williams DA. Interventional pain medicine: retreat from the biopsychosocial model of pain. Trans/ Behav Med 2012;2:106-16.

8 Sivaganesan A, Chotai S, Parker SL, et al. Predictors of the efficacy of epidural steroid injections for structural lumbar degenerative pathology. Spine J 2016;16:928-34.

9 Kennedy DJ, Engel A, Kreiner DS, et al. Fluoroscopically guided diagnostic and therapeutic intra-articular sacroiliac joint injections: a systematic review. Pain Med 2015;16:1500-18.

10 Şencan $\mathrm{S}$, Çelenlioğlu AE, Asadov R, et al. Predictive factors for treatment success of transforaminal epidural steroid injection in lumbar disc herniation-induced sciatica. Turk J Med Sci 2020;50:126-31.

11 Cohen S, Doshi T, Dawson T, et al. Prognostic value of hypersensitivity reactions on epidural steroid injection outcomes: a phenotypic signature? A prospective cohort study. Reg Anesth Pain Med 2019;44:586-94.
12 Cohen SP, Bhaskar A, Bhatia A, et al. Consensus practice guidelines on interventions for lumbar facet joint pain from a Multispecialty, International Working group. Reg Anesth Pain Med 2020;45:424-67.

13 Thawrani DP, Agabegi SS, Asghar F. Diagnosing Sacroiliac joint pain. J Am Acad Orthop Surg 2019;27:85-93.

14 Cohen SP, Bicket MC, Jamison D, et al. Epidural steroids: a comprehensive, evidencebased review. Reg Anesth Pain Med 2013;38:175-200.

15 Cohen SP, Bicket MC, Kurihara C, et al. Fluoroscopically guided vs Landmark-Guided Sacroiliac joint injections: a randomized controlled study. Mayo Clinic Proceedings 2019;94:628-42

16 Cohen SP, Doshi TL, Constantinescu OC. Effectiveness of lumbar facet joint blocks and their predictive value before radiofrequency denervation: the facet treatment study (facts), a randomized, controlled clinical trial. Anesthesiology 2018;129:521-46.

17 Cohen SP, Mao J, Vu T-N, et al. Does pain score in response to a standardized subcutaneous local anesthetic injection predict epidural steroid injection outcomes in patients with lumbosacral radiculopathy? A prospective correlational study. Pain Med 2013; 14:327-35.

18 Jamison RN, VadeBoncouer T, Ferrante FM. Low back pain patients unresponsive to an epidural steroid injection: identifying predictive factors. Clin J Pain 1991;7:311-7.

19 Szadek KM, van der Wurff $P$, van Tulder MW, et al. Diagnostic validity of criteria for sacroiliac joint pain: a systematic review. J Pain 2009;10:354-68.

20 Eklund A, De Carvalho D, Pagé I, et al. Expectations influence treatment outcomes in patients with low back pain. A secondary analysis of data from a randomized clinical trial. Eur J Pain 2019;23:1378-89.

21 Dworkin RH, Turk DC, Wyrwich KW, et al. Interpreting the clinical importance of treatment outcomes in chronic pain clinical trials: IMMPACT recommendations. J Pain 2008;9:105-21.

22 Jakobsen JC, Ovesen C, Winkel P, et al. Power estimations for non-primary outcomes in randomised clinical trials. BMJ Open 2019;9:e027092

23 Austin PC, Steyerberg EW. The number of subjects per variable required in linear regression analyses. J Clin Epidemiol 2015;68:627-36.

24 Cohen SP, Vase L, Hooten WM. Chronic pain: an update on burden, best practices, and new advances. Lancet 2021;397:2082-97.

25 Wilkens P, Scheel IB, Grundnes 0, et al. Prognostic factors of prolonged disability in patients with chronic low back pain and lumbar degeneration in primary care: a cohort study. Spine 2013;38:65-74

26 Aljawadi A, Sethi G, Islam A, et al. Sciatica presentations and predictors of poor outcomes following surgical decompression of herniated lumbar discs: a review article. Cureus 2020;12:e11605.

27 Harris I, Mulford J, Solomon M, et al. Association between compensation status and outcome after surgery: a meta-analysis. JAMA 2005;293:1644-52.

28 Pakpour AH, Yaghoubidoust M, Campbell P. Persistent and developing sleep problems: a prospective cohort study on the relationship to poor outcome in patients attending a pain clinic with chronic low back pain. Pain Pract 2018;18:79-86.

29 Collados-Maestre I, Lizaur-Utrilla A, Martinez-Mendez D, et al. Concomitant low back pain impairs outcomes after primary total knee arthroplasty in patients over 65 years: a prospective, matched cohort study. Arch Orthop Trauma Surg 2016;136:1767-71.

30 Scherer M, Hansen H, Gensichen J, et al. Association between multimorbidity patterns and chronic pain in elderly primary care patients: a cross-sectional observational study. BMC Fam Pract 2016;17:68.

31 Bialosky JE, Bishop MD, Cleland JA. Individual expectation: an overlooked, but pertinent, factor in the treatment of individuals experiencing musculoskeletal pain. Phys Ther 2010;90:1345-55.

32 Hafkamp FJ, Gosens T, de Vries J, et al. Do dissatisfied patients have unrealistic expectations? A systematic review and best-evidence synthesis in knee and hip arthroplasty patients. EFORT Open Rev 2020;5:226-40.

33 Engle AM, Chen Y, Marascalchi B. Precipitating events associated with lumbar radicular pain and their association with epidural steroid injection outcomes. Pain Med 2019:20:2360-70.

34 Odonkor CA, Chen Y, Adekoya $\mathrm{P}$, et al. Inciting events associated with lumbar facet joint pain. Anesth Analg 2018;126:280-8

35 Campbell CM, Buenaver LF, Raja SN, et al. Dynamic pain phenotypes are associated with spinal cord stimulation-induced reduction in pain: a repeated measures observational pilot study. Pain Med 2015;16:1349-60.

36 Schiff E, Eisenberg E. Can quantitative sensory testing predict the outcome of epidura steroid injections in sciatica? A preliminary study. Anesth Analg 2003;97:828-32.

37 Maher DP, Ding W, Singh S, et al. Thermal QST phenotypes associated with response to lumbar epidural steroid injections: a pilot study. Pain Med 2017;18:1455-63.

38 Thawrani DP, Agabegi SS, Asghar F. Diagnosing Sacroiliac joint pain. J Am Acad Orthop Surg 2019:27:85-93.

39 Karran EL, McAuley JH, Traeger AC, et al. Can screening instruments accurately determine poor outcome risk in adults with recent onset low back pain? A systematic review and meta-analysis. BMC Med 2017:15:13.

40 Carreon $L Y$, Bratcher KR, Ammous F, et al. Cost-Effectiveness of lumbar epidural steroid injections. Spine 2018:43:35-40.

41 Peile E. Evidence-based medicine and values-based medicine: partners in clinical education as well as in clinical practice. BMC Med 2013:11:40. 